\title{
ON DERIVATION ALGEBRAS OF MALCEV ALGEBRAS
}

\author{
ERNEST L. STITZINGER
}

\begin{abstract}
It is shown that if $A$ is a Malcev algebra over a field of characteristic 0 , then $A$ is semisimple if and only if the derivation algebra $\mathscr{D}(A)$ is semisimple. It is then shown that $A$ is semisimple if and only if $A^{*}=\mathfrak{L}(A)+\mathfrak{D}(A)$ is semisimple, where $\mathfrak{L}(A)$ is the Lie multiplication algebra of $A$.
\end{abstract}

Let $A$ be a nonassociative algebra over a field of characteristic 0 and let $\mathfrak{D}(A)$ be the derivation algebra of $A$. For certain classes of algebras (Lie, Jordan, alternative), there are results linking the semisimplicity of $A$ to that of $\mathfrak{D}(A)$. Although some results of a related nature are available for Malcev algebras, it has not yet been shown that the semisimplicity of $A$ and $\mathfrak{D}(A)$ are equivalent. It is the purpose of the present note to obtain this result and several related ones.

All algebras discussed here will be finite dimensional over a field of characteristic 0 .

TheOREM 1. Let $A$ be a Malcev algebra over a field $F$ of characteristic 0 . Then $A$ is semisimple if and only if $\mathfrak{D}(A)$ is semisimple.

Proof. Suppose that $\mathfrak{D}(A)$ is semisimple. By [11, Theorem 1] the radical $R(A)$ of $A$ is contained in the center $Z(A)$ of $A$. Then by [10, Theorem 1] $A$ is the direct sum of $R(A)$ and a maximal semisimple subalgebra $S(A)$ of $A$. Then $\mathfrak{D}(A)$ is the direct sum of ideals $\mathfrak{D}_{1}$ and $\mathfrak{D}_{2}$ where

$$
\mathfrak{D}_{1}=\{D \in \mathfrak{D}(A) ; D: S(A) \rightarrow S(A), D: R(A) \rightarrow 0\}
$$

and

$$
\mathfrak{D}_{2}=\{D \in \mathfrak{D}(A) ; D: S(A) \rightarrow 0\}
$$

(see the proof of [11, Theorem 1]). Suppose that $R(A) \neq 0$. Let $T$ be the projection of $A$ onto $R(A)$ with null space $S(A)$. Since $0 \neq R(A) \subseteq Z(A), T$ $\in \mathfrak{D}_{2}$ and $T \neq 0$. Let $T_{1} \in \mathfrak{D}_{2}, x \in R(A)$ and $y \in S(A)$. Then, since $R(A)$ is $\mathfrak{D}(A)$-invariant by $[3$, Theorem 14$]$,

Received by the editors December 17, 1975 and, in revised form, June 8, 1976.

AMS (MOS) subject classifications (1970). Primary 17A30. 


$$
(x+y)\left[T, T_{1}\right]=(x+y) T T_{1}-(x+y) T_{1} T=x T_{1}-x T_{1}=0 .
$$

Hence $T \in Z\left(\mathscr{D}_{2}\right)$ and since $\mathscr{D}_{1}$ and $\mathfrak{D}_{2}$ are ideals in $\mathscr{D}(A), T \in Z(\mathscr{D}(A))$. This contradicts the semisimplicity of $\mathfrak{D}(A)$, hence $R(A)=0$.

Conversely let $K$ be an algebraic closure of $F$. Then $\mathfrak{D}\left(A_{K}\right) \simeq \mathscr{D}(A)_{K}$ (see [6, p. 233]). Semisimplicity of a Malcev algebra is equivalent to nondegeneracy of the Killing form [5, Theorem $\mathrm{A}]$ and the latter is preserved under extension of the base field, hence $A_{K}$ is semisimple. By [5, Korollar 1], $A_{K}$ is the direct sum of simple ideals. Each of these simple ideals is either a simple Lie algebra or is a 7 dimensional algebra obtained from a Cayley algebra as in [8, pp. 433-435]. This follows from combined results of Sagle [9] and Loos [5, Theorem B]. In either case, the simple algebra has simple derivation algebra. In particular, the non-Lie simple algebra has the exceptional simple Lie algebra $G_{2}$ for its derivation algebra [8, p. 455]. It follows that $\mathfrak{D}\left(A_{K}\right)$ is semisimple, hence $\mathfrak{D}(A)$ is also.

In order to extend a classical result of Leger and Togo [4] to general algebras, Ravisankar [6] considered $A^{*}=\mathfrak{L}(A)+\mathfrak{D}(A)$ where $\mathfrak{R}(A)$ is the Lie multiplication algebra of $A . \mathfrak{L}(A)$ is an ideal of $A^{*}[6$, p. 225]. We now show that the result of Theorem 1 holds with $\mathfrak{D}(A)$ replaced by $A^{*}$. In order to do this we need the following result.

LeMma. Let $A$ be a Malcev algebra over a field of characteristic 0 . Then $\mathfrak{R}(A)$ is semisimple or 0 if and only if $R(A) \subseteq Z(A)$.

Proof. If $R(A) \subseteq Z(A)$, then $R(A)$ is complemented by a semisimple subalgebra $S(A)$ by [10, Theorem 1]. If $S(A)=0$, then $\mathfrak{R}(A)=0$. Suppose that $S(A) \neq 0$. Then $S(A)$ is $\mathfrak{R}(A)$ invariant since $R(A) \subseteq Z(A)$, and the restriction mapping is an isomorphism from $\mathfrak{R}(A)$ to $\mathfrak{R}(S(A))$. Since $S(A)$ is semisimple, $\mathfrak{L}(S(A))$ is also [8, Corollary 7.3].

Conversely if $\mathfrak{L}(A)$ is semisimple, then, using a theorem of Weyl [1, Théorème 2, p. 75], $Z(A)$ is complemented by an ideal $B$ of $A$. Clearly $Z(B)=0$ and $\mathfrak{L}(A) \simeq \mathfrak{E}(B)$. Hence $B$ is semisimple by [8, Theorem 7.2] and the result holds. If $\mathfrak{L}(A)=0$, then $Z(A)=A$.

THEOREM 2. Let $A$ be a Malcev algebra over a field of characteristic 0 . Let $A^{*}=\mathfrak{L}(A)+\mathfrak{D}(A)$. Then $A^{*}$ is semisimple if and only if $A$ is semisimple.

Proof. If $A$ is semisimple, then $\mathscr{D}(A)$ and $\mathfrak{L}(A)$ are semisimple. Then $A^{*} / \mathfrak{L}(A) \simeq \mathfrak{D}(A) / \mathfrak{D}(A) \cap \mathfrak{L}(A)$ is semisimple by [1, Corollaire 2, p. 76]. Since $R\left(A^{*}\right)$ projects onto the radical of $A^{*} / \mathfrak{R}(A)$ [1, Corollaire 3, p. 76], $R\left(A^{*}\right)$ $\subseteq \mathfrak{L}(A)$. Hence $R\left(A^{*}\right)=0$ and $A^{*}$ is semisimple.

Conversely, if $A^{*}$ is semisimple, then $\mathfrak{L}(A)$ is semisimple or $\mathfrak{R}(A)=0$, hence $R(A) \subseteq Z(A)$ and $R(A)$ is complemented by a semisimple subalgebra $S(A)$. Suppose $R(A) \neq 0$. By [11, Theorem 1], $\mathscr{D}(A)$ acts completely reducibly on $A$, hence $\mathfrak{D}(A)=S \oplus Z$ where $S$ is a semisimple subalgebra of $\mathscr{D}(A)$ and $Z$ is the center of $\mathfrak{D}(A)$. Since $A^{*} / \mathfrak{L}(A)$ is semisimple, $Z \subseteq \mathfrak{L}(A)$. Let $T$ be the projection of $A$ on $R(A)$ with null space $S(A)$. Then $T \in \mathscr{D}(A)$. Let $\mathscr{D}_{1}$ and $\mathscr{D}_{2}$ 
be as in the proof of Theorem 1. Then both $R(A)$ and $S(A)$ are invariant under $\mathfrak{D}_{1} \oplus \mathfrak{D}_{2}=\mathfrak{D}(A)$, hence $T$ commutes with each element of $\mathfrak{D}(A)$. Therefore $T \in Z \subseteq \mathfrak{L}(A)$. Since $R(A) \subseteq Z(A), \mathfrak{L}(A)$ annihilates $R(A)$. Hence $T=0$ and $R(A)=0$.

\section{REFERENCES}

1. N. Bourbaki, Eléments de mathématique, Livre XXVI, Groupes et algèbres de Lie, Chap. I, Actualités Sci. Indust., no. 1285, Hermann, Paris, 1960. MR 24 \# A2641.

2. W. H. Davenport, On inner derivations of Malcev algebras, Rocky Mountain J. Math. 2 (1972), 565-568. MR 46 \#9129.

3. E. N. Kuz'min, Mal'cev algebras and their representations, Algebra i Logika 7 (1968), 48-69 $=$ Algebra and Logic 7 (1968), 233-244. MR 40 \#5688.

4. G. F. Leger, Jr. and S. Tôgô, Characteristically nilpotent Lie algebras, Duke Math. J. 26 (1959), 623-628. MR 22 \#5659.

5. O. Loos, Uber eine Beziehung zwischen Malcev-Algebren und Lie-Triplesystemen, Pacific J. Math. 18 (1966), 553-562. MR 33 \# 7385.

6. T. S. Ravisankar, Characteristically nilpotent algebras, Canad. J. Math. 23 (1971), 222-235. MR 43 \#2031.

7. - On Malcev algebras, Pacific J. Math. 42 (1972), 227-234. MR 47 \# 1905.

8. A. A. Sagle, Malcev algebras, Trans. Amer. Math. Soc. 101 (1961), 426-458. MR 26 \# 1343.

9. , Simple Malcev algebras over fields of characteristic zero, Pacific J. Math. 12 (1962), 1057-1078. MR 27 \# 184.

10. E. L. Stitzinger, Malcev algebras with $J_{2}$-potent radical, Proc. Amer. Math. Soc. 50 (1975), 1-9. MR 51 \# 10424.

11. On derivation algebras of Malcev algebras and Lie triple systems, Proc. Amer. Math. Soc. 55 (1976), 9-13.

Department of Mathematics, North Carolina State University, Raleigh, North CaroLINA 27607 